\title{
Editoriais Exclusivos e Artigos de Maior Sucesso da RBCI
}

$\mathrm{F}$ inalizamos o ano de 2007 com outra edição da Revista Brasileira de Cardiologia Invasiva (RBCI), que publica, entre outros, artigos originais premiados no XXIX Congresso da Sociedade Brasileira de Hemodinâmica e Cardiologia Intervencionista $(\mathrm{SBHCl})$, artigos de revisão que analisam com profundidade os tópicos propostos, relatos de caso e imagens de doenças raras, e também editoriais especiais que discutem e ampliam o conhecimento dos temas apresentados.

O editorial redigido pelo grupo da Cardiovascular Research Foundation/Columbia University Medical Center, em Nova York, Estados Unidos, liderado pelo dr. Martin B. Leon, avalia os artigos dos drs. Fernando de Martino et al., do InCor, e Marco Aurélio de Magalhães et al., do Hospital Israelita Albert Einstein, a respeito do controvertido tema do emprego dos stents farmacológicos no tratamento do infarto agudo do miocárdio.

Já o dr. Paolo Angelini, do Texas Heart Institute, em Houston, Estados Unidos, comenta a rara síndrome de Bland-White-Garland, que, por curioso acaso, fez com que comunicações das formas infantil e adulta, provenientes de diferentes instituições (Faculdade de Medicina de Botucatu e Instituto de Cardiologia do Rio Grande do Sul, respectivamente), chegassem à RBCI em curto espaço de tempo, permitindo a oportunidade única de criteriosa análise do tema por uma autoridade reconhecida em cardiopatias congênitas.

O dr. José Antonio Marin-Neto introduz e motiva os leitores a mergulharem no artigo de revisão "Aspectos Metodológicos Relacionados aos Sistemas Manométricos Utilizados em Estudos Hemodinâmicos", do dr. Edson A. Bregagnollo, que trata de importantes aspectos técnicos envolvidos na mensuração das pressões intravasculares e intracardíacas, menos valorizados na atualidade pelas novas gerações de cardiologistas intervencionistas.

Essa lista de excelência traz ainda o editorial do dr. Protásio Lemos da Luz, que tece considerações sobre o artigo original do dr. Wilson Pimentel Filho et al., a respeito do tratamento da doença coronária crônica.

E o excelente artigo do dr. Domingo M. Braile et al., que trata da importância da indexação para as revistas científicas, coroa os editoriais. Entre as múltiplas atribuições do dr. Braile, um dos ícones da cirurgia cardíaca brasileira, está a de editor da Revista Brasileira de Cirurgia Cardiovascular, cujo destino vem sendo conduzido com sucesso nos últimos seis anos, tendo sido recentemente indexada no PubMed/MEDLINE, demonstrando que a determinação, a persistência e o trabalho de grupo levaram a publicação oficial da Sociedade Brasileira de Cirurgia Cardiovascular a alcançar o patamar científico desejado pelas melhores revistas médicas. Esperamos que esse artigo, lido e relido inúmeras vezes por esta editora, na tentativa de absorver todos os seus ensinamentos, sirva de inspiração aos nossos diretores, co-editores, autores e leitores, na busca dessa mesma meta para a RBCI.

Com o intuito de procurar o melhor para esta publicação, contamos, desde 9 de agosto de 2007, com uma ferramenta em nosso website que contabiliza o número de acessos e downloads de cada artigo publicado desde a edição 11.3. Em última análise, permite avaliar os temas que despertam em você, leitor, maior interesse, ajudando, assim, a redirecionar o curso editorial sempre que necessário.

Editores e diretores sentem-se gratificados ao constatar o número crescente de visitas ao website da $\mathbf{R B C I}$ ao longo dos meses, totalizando nesse período de avaliação mais de 10 mil arquivos gerados em PDF (Figura 1).

Surpreendeu-nos o grande interesse causado pelos artigos originais, que rivalizam em procura com os artigos de revisão, respectivamente em segundo e primeiro lugares (Figura 2), fato que reforçará os planos de mudança da linha editorial para 2008. Procuraremos alcançar a meta de $75 \%$ de artigos originais por edição, requeridos pelo SciELO para a indexação da $\mathbf{R B C I}$.

Notamos também que o acesso às últimas três edições do ano de 2007 pelo menos triplicou a média alcançada pelas edições prévias (Figura 3), outra vez demonstrando que estamos em sintonia com o interesse de nossos leitores.

Avaliamos também os artigos de maior procura das edições mais consultadas até 25 de novembro de

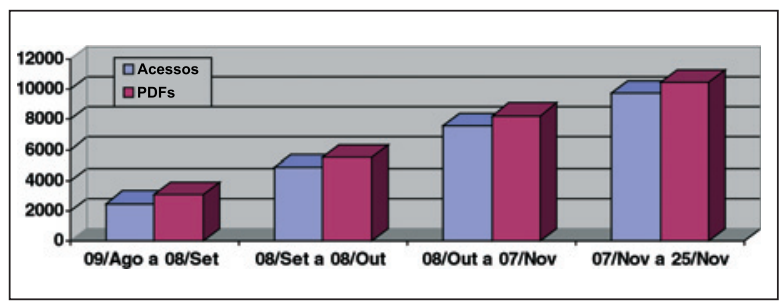

Figura 1 - Freqüência acumulada de acessos e downloads da $\mathbf{R B C I}$ até 25 de novembro de 2007. 


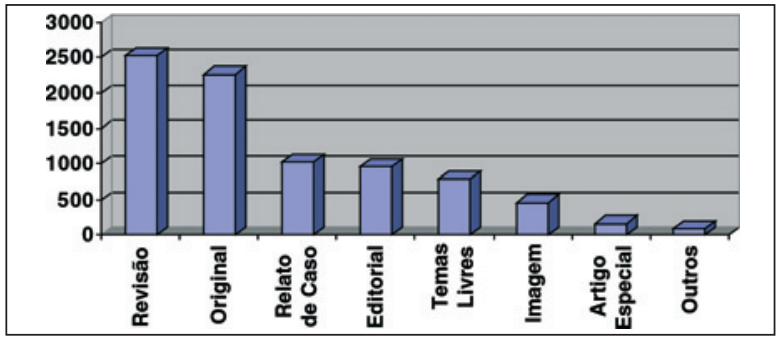

Figura 2 - Seções da $\mathbf{R B C l}$ mais acessadas.

2007 (Tabela). O artigo que maior interesse gerou, nesse período de avaliação, foi "Cardiomiopatia de Takotsubo como Causa de Infarto Agudo do Miocárdio em Paciente em Uso de Anti-Histamínico Associado a Pseudo-Efedrina", de José Armando Mangione e Gustavo Ithamar Souto Maior, do Hospital Beneficência Portuguesa de São Paulo. Adicionalmente, ficamos impressionados com a procura dos artigos ligados à intervenção extracardíaca, o que mostra que a RBCI é importante canal de veiculação dessa nova área da Medicina, a Terapia Endovascular. Esperamos que esse fato inspire os dirigentes da Sociedade Brasileira de Radiologia Intervencionista e Cirurgia Endovascular (SOBRICE) a alcançar, junto com a $\mathrm{SBHCl}$, um denomi-

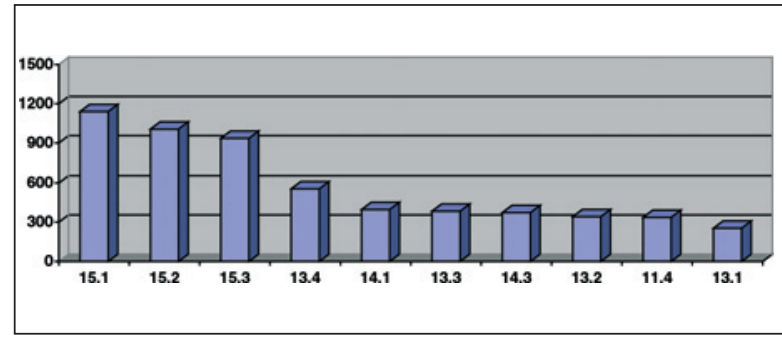

Figura 3 - Edições da $\mathbf{R B C l}$ mais acessadas.

nador comum no desenvolvimento dessa nova área de atuação.

Por fim, gostaria de agradecer ao presidente da $\mathrm{SBHCl}$, dr. Luiz Alberto Mattos, ao diretor de comunicações, dr. Rogério Sarmento-Leite, aos co-editores drs. Carlos Augusto Pedra e Marco Wainstein, à assessora editorial Rosangela Monteiro, à revisora Elena Mattos, à gerente da $\mathrm{SBHCl}$ Norma Cabral, à secretária de publicações Natalia Mariotto, e ao designer em artes gráficas da $\mathrm{SBHCl}$ Gabriel Marques, o apoio e a inestimável dedicação ao longo de 2007 na condução da RBCI.

Áurea J. Chaves Editora

TABELA

Artigos de maior sucesso das edições mais consultadas até 25 de novembro de 2007

\begin{tabular}{|c|c|c|c|c|}
\hline & & & Visitas & PDFs gerados \\
\hline 15.3 & \multirow{2}{*}{ Editorial } & \multicolumn{3}{|l|}{ O Ensaio Clínico COURAGE: Visão de um Cardiologista Clínico } \\
\hline $\begin{array}{l}\text { Cardiologia } \\
\text { Invasiva }\end{array}$ & & G. Feitosa & 234 & 240 \\
\hline 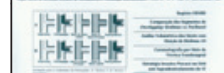 & \multirow[t]{2}{*}{ Editorial } & Uma Nova Geração de Stents Farmacológicos & & \\
\hline$\Delta \Delta \Delta \Delta=$ & & R. Sarmento-Leite & 151 & 155 \\
\hline 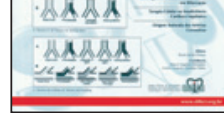 & Editorial & $\begin{array}{l}\text { Ensinando a Técnica de Sones: Necessidade ou Tradição? } \\
\text { C. Rodrigues Alves, V. Lima }\end{array}$ & 149 & 150 \\
\hline \multirow{8}{*}{\begin{tabular}{|l}
15.2 \\
Cardiologia \\
Invasiva \\
\\
\end{tabular}} & \multirow[t]{3}{*}{ Original } & \multicolumn{3}{|l|}{ Oclusão Percutânea do Canal Arterial > 3 mm com Auxílio do Biótomo } \\
\hline & & \multirow{2}{*}{$\begin{array}{l}\text { D. Arnoni, J. Peña, V. Fontes, S. Braga, C. Esteves, W. Ferreira, } \\
\text { S. Pedra, M. Santana, J. Santin, J. Silva, R. O'Connor, C. Pedra }\end{array}$} & & \\
\hline & & & 256 & 259 \\
\hline & \multirow[t]{2}{*}{ Original } & \multicolumn{3}{|l|}{$\begin{array}{l}\text { Eficácia e Segurança do Cateter "Pronto" para Aspiração Manual } \\
\text { de Trombo Durante Angioplastia Primária no Infarto } \\
\text { Agudo do Miocárdio com Elevação do Segmento ST }\end{array}$} \\
\hline & & M. Santos, C. Fonseca, M. Godoy & 223 & 232 \\
\hline & \multirow[t]{3}{*}{$\begin{array}{l}\text { Relato } \\
\text { de Caso }\end{array}$} & $\begin{array}{l}\text { Tratamento de Trombose Tardia de Stent Farmacológico } \\
\text { com Cateter Extrator de Trombo }\end{array}$ & & \\
\hline & & M. Lacativa, L. Oliveira, R. Cardoso, J. Frighetto, R. Cardoso, & & \\
\hline & & J. Campos, F. Cardoso, L. Alves & 132 & 148 \\
\hline
\end{tabular}




\section{CONTINUAÇÃO - TABELA}

Artigos de maior sucesso das edições mais consultadas até 25 de novembro de 2007

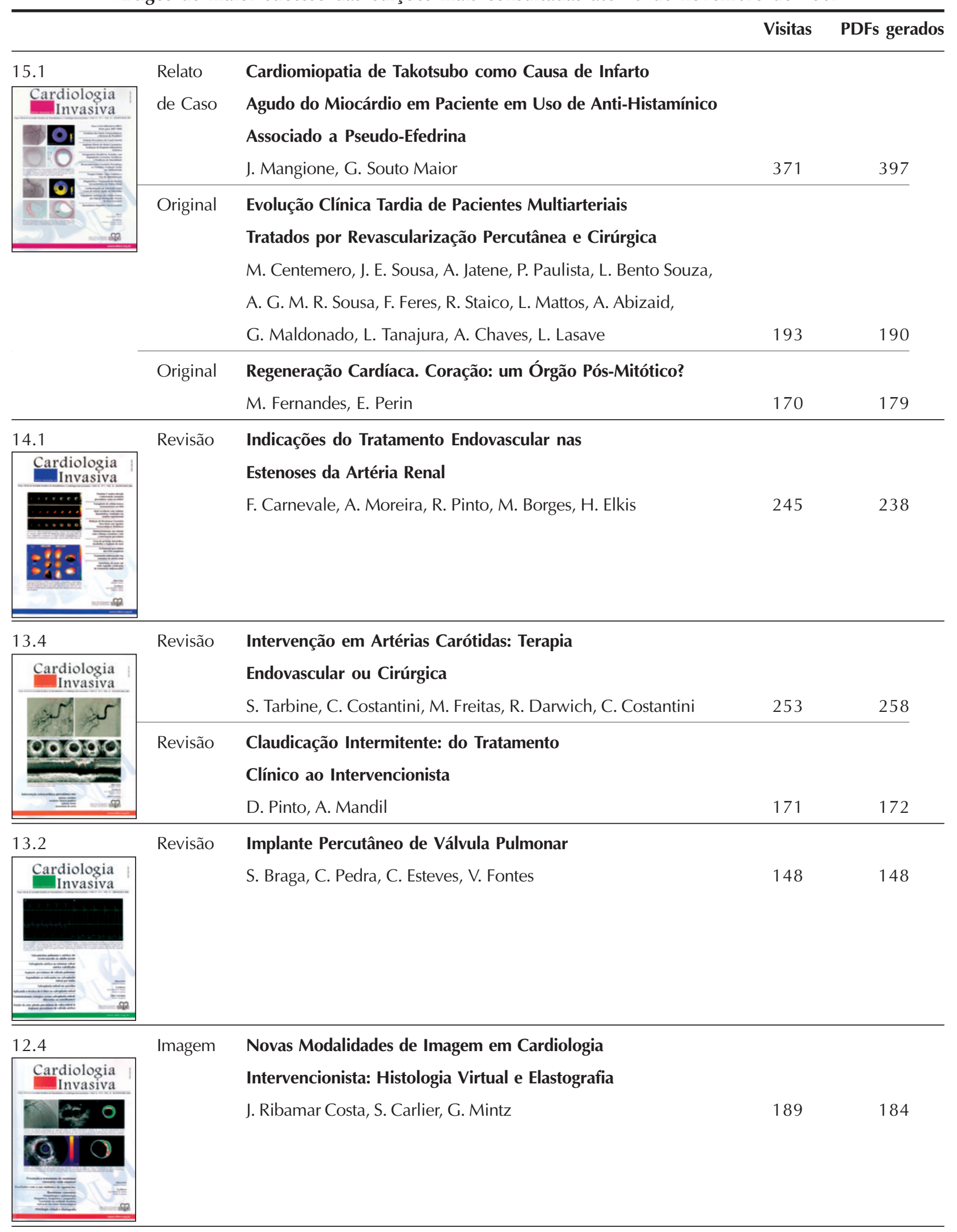

